\title{
Fixturing Options for Atom Probe Tomography
}

Katherine Rice ${ }^{1}$, Yimeng Chen ${ }^{1}$, Robert Ulfig ${ }^{1}$ and Tsuyoshi Onishi ${ }^{2}$

${ }^{1}$ CAMECA Instruments, Inc., Madison, Wisconsin, United States, ${ }^{2}$ Hitachi High-Tech Corporation, Tokyo, Tokyo, Japan

Atom probe tomography (APT) is a powerful technique to obtain 3D compositional information on the nanoscale. As with many microscopy techniques, specimen preparation is critical to achieving highquality data and specimen yield. Strategies for focused ion beam (FIB) based atom probe specimen preparation were introduced in the early 2000's and its application has been broadened to accommodate various types of fixtures that allow correlative analyses [1,2]. Correlative microscopy on APT specimens provides additional specimen characterization that can help target specific regions of interest during the specimen preparation process or inform the reconstructed data. Here we detail CAMECA's offerings to assist in FIB-based specimen preparation.

Wire-based specimens can be used in both LEAPTM and EIKOS ${ }^{\mathrm{TM}}$ atom probe platforms and are ideal for correlative microscopy techniques such as TEM and electron tomography. CAMECA ${ }^{\circledR}$ offers a wide variety of accessories to facilitate specimen preparation, including wire-based specimen holders that can accommodate up to six wires for either preparing flat substrates or mounting FIB based specimens or microtip coupon holders. The holders are also QuickLoader ${ }^{\mathrm{TM}_{-}}$and Ferrovac Direct-Docking compatible, not requiring a separate shuttle to be used to load into a Thermo Scientific ${ }^{\mathrm{TM}}$ stage. Pin-mounted wire specimen holders are also available for standard 3.5mm SEM stage use.

Atom probe specimens can also be mounted to half-grid substrates to facilitate TEM/APT correlative work. CAMECA, in collaboration with Hitachi High-Tech Corporation, have developed FIB-ready Sibased half grid substrates with rectangular posts that have an end dimension of $3 \times 3$ microns. Shown in Fig 1a and $\mathrm{b}$, these grids need no pre-preparation to be used as APT mounting substrates. Five grid posts are available per half-grid, enabling multiple specimens to be prepared per liftout. Protective 'side wings' assist in handling to minimize the risk of damaging prepared specimens.

In this work, solutions to correlative microscopy analysis using specialized fixtures offered by CAMECA will be demonstrated. Application examples suited to microtip coupon stubs or wire-mounted stubs for STEM and transmission EBSD analysis will be presented. The benefit of using stub-mounted coupons or wires in terms of minimizing transferring time will be discussed.

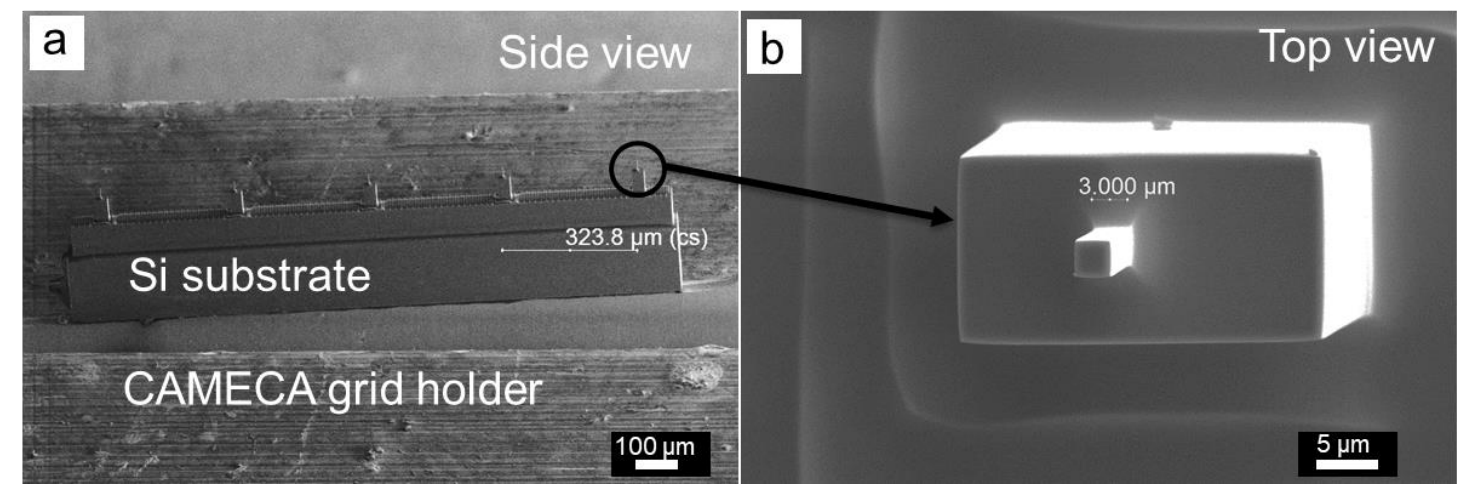

Figure 1. a) Side view of NanoMesh-APT showing grid posts available for FIB liftout mounting; b) Top view of a single Si grid post showing area for mounting a FIB liftout. 


\section{References}

[1] Larson, D. J., et al. "Focused ion-beam specimen preparation for atom probe field-ion microscopy characterization of multilayer film structures." Nanotechnology 10.1 (1999): 45.

[2] Gorman, Brian P., et al. "Hardware and techniques for cross-correlative TEM and atom probe analysis." Microscopy Today 16.4 (2008): 42-47. 